\title{
Dry Digital Gangrene
}

Trusha Shah, OMS III; Jay H. Shubrook, DO

From the Touro University

College of Osteopathic

Medicine-CA in Vallejo.

Disclaimer: Dr Shubrook, an associate editor of The Journal of the American Osteopathic Association, was not involved

in the editorial review or decision to publish this article.

Financial Disclosures: None reported.

Support: None reported.

Address correspondence to Jay H. Shubrook, DO, 1310 Club Dr, Mare Island, Vallejo, CA $94592-1187$

E-mail: jay.shubrook@tu.edu

Submitted July 14, 2018;

accepted July 27, 2018.

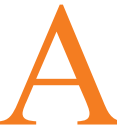

61-year-old man presented to a clinic with the complaint of 6 weeks of right middle finger pain and swelling. His medical history included type 2 diabetes mellitus, hypertension, peripheral arterial occlusive disease, and chronic kidney disease. The patient received dialysis through an arteriovenous fistula in his right arm. The right middle finger became gangrenous after 8 weeks (image A). Postpresentation arteriogram records indicated steal syndrome causing vascular insufficiency in all right hand digits (image $\mathbf{B}$ ). The right middle finger autoamputated a few weeks later.

Hemodialysis is a common risk factor for ischemia of the upper extremity, and amputation is a common sequelae. ${ }^{1}$ Studies have shown that currently the best management of dialysis-associated steal syndrome is distal revascularization with interval ligation, which involves ligating an artery distal to the fistula and subsequently forming a bypass. $^{2}$ Owing to the high mortality rates of dialysis patients who require upper extremity amputations, early revascularization efforts are key. ${ }^{3}$ (doi:10.7556/jaoa.2018.166)

\section{References}

1. Ferraresi R, Acuña-Valerio J, Ferraris M, et al. Angiographic study of upper limb vascularization in a large cohort of hemodialysis patients with critical hand ischemia. Minerva Cardioangiol. 2016;64(6):642-647.

2. Regalado S, Navuluri R, Vikingstad E. Distal revascularization and interval ligation: a primer for the vascular and interventional radiologist. Semin Intervent Radiol. 2009;26(2):125-129. doi:10.1055/s-0029-1222456

3. Stone AV, Xu NM, Patterson RW, Koman LA, Smith BP, Li Z. Five-year mortality for patients with end-stage renal disease who undergo upper extremity amputation. J Hand Surg Am. 2015;40 (4):666-672. doi:10.1016/j.jhsa.2014.12.036

๑) 2018 American Osteopathic Association

Keywords: diabetes mellitus, gangrene, hemodialysis
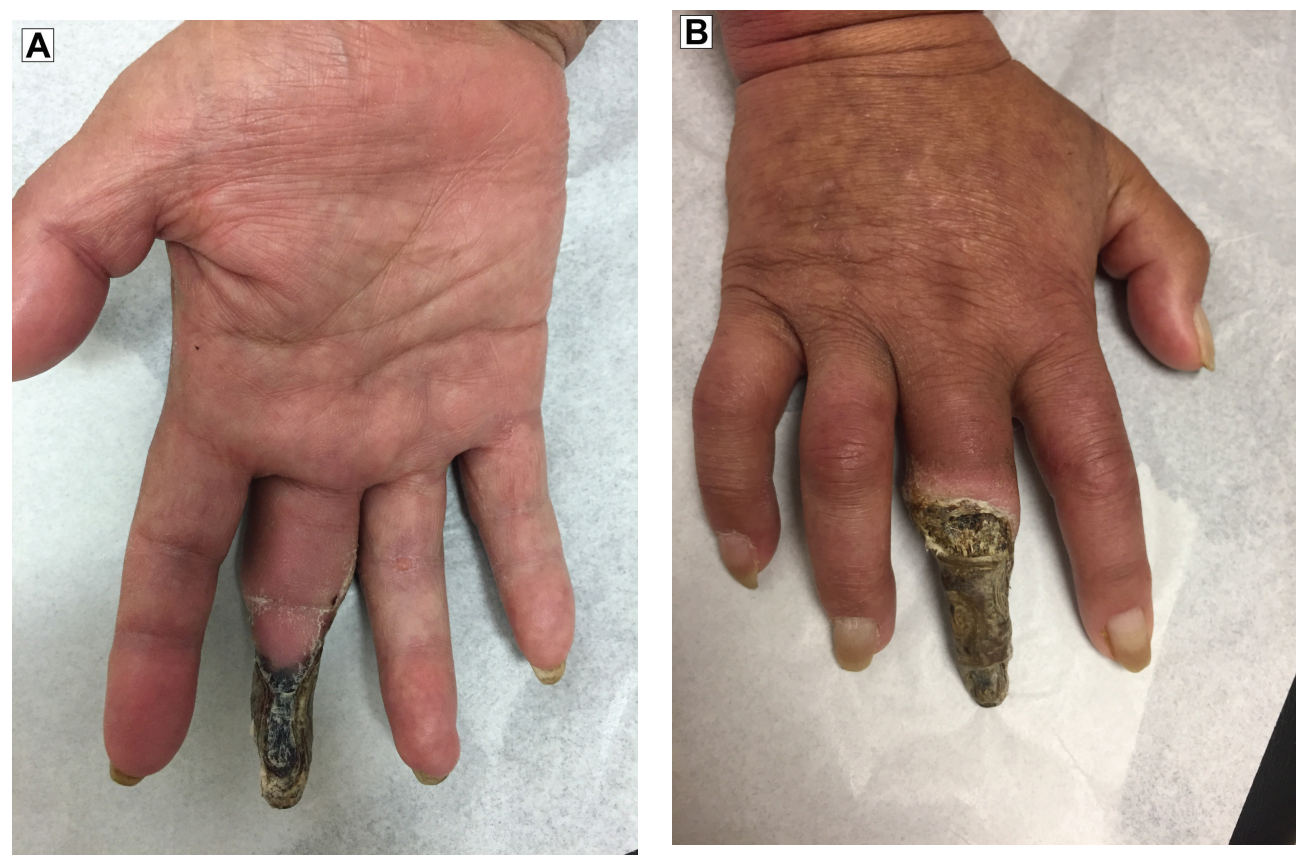